\title{
PROFIT DISTRIBUTION IN IPD PROJECTS BASED ON WEIGHT FUZZY COOPERATIVE GAMES
}

\author{
Shuwen GUO®1,2*, Junwu WANG®1 \\ ${ }^{1}$ School of Civil Engineering and Architecture, Wuhan University of Technology, Wuhan, China \\ ${ }^{2}$ Evergrande School of Management, Wuhan University of Science and Technology, Wuhan, China
}

Received 24 January 2021; accepted 3 November 2021; first published online 31 December 2021

\begin{abstract}
Integrated Project Delivery (IPD) is regarded as an effective project delivery method that can deal with the challenge of the rapid development of the architecture, engineering, and construction (AEC) industry. In the IPD team, the alliance profit is not distributed fairly and effectively due to uncertainty, preventing the achievement of the IPD project goals. This study focuses on optimizing the profit distribution among stakeholders in IPD projects and uses quadratic programming models to solve fuzzy cooperative games in the IPD. A payoff function is used in the fuzzy alliance to determine the characteristics of the interval-valued fuzzy numbers, and different weights of the alliance and the efficiency of the player's participation in the IPD are considered in the profit distribution. A case study is conducted, and the results of the proposed method are compared with those of crisp cooperative games. The results show that the fuzzy cooperative game increases the profit of participants in IPD projects. It is more practical to use weight fuzzy cooperative games than crisp games to express imputation. Moreover, the quadratic programming models and methods result in a fair and efficient profit distribution scheme in IPD projects.
\end{abstract}

Keywords: profit distribution, integrated project delivery, fuzzy cooperative game, weight of alliance, interval-valued fuzzy numbers, quadratic programming.

\section{Introduction}

As the AEC industry has grown, the more complex and expensive, traditional project organization has caused cause waste, increased cost and time, and led to more adversarial relationships, resulting in projects often failing to deliver the results that owners expect. Traditional project design and construction delivery methods are segmental. Tradition projects involve "siloed" structures with rigid hierarchies. Construction Management Association of America [CMAA] (2010), Ernst and Young (2014) surveyed 365 industrial projects and found that about $64 \%$ of them were faced with the cost overrun and $73 \%$ were delayed. Sixty-five per cent of project failures are due to soft aspects, such as personnel, organization, and governance. Complex and large-scale projects are characterized by substantial uncertainty, complexity, multiple work processes, and the interaction of project participants.

In recent years, the emergence of relational project delivery arrangements (IPD and alliance contracts) has represented a paradigm shift because risk-sharing rather than risk transfer occurs, which changes the traditional roles and relationships of the key stakeholders in the projects. This occurrence has increased project team cooperation and integration, optimized the use of various resources of the project, and changed the fragmented state of each stage in the traditional delivery mode. Thus, the project delivery concept has emerged, creating a high level of trust and open communication between participants essential for the success of the project. Participants in IPD projects (i.e., owners, architects, and builders) have estimated the value provided by IPD, including efficiency, cost management, superior results, and flexibility (Boodai, 2014). IPD encourages all participants to maximize project performance through positive behaviors. By eliminating behaviors that hinder creativity and collaboration, the goals of each participant are aligned with the project goals, and actions that add value to the project are encouraged (Ashcraft, 2012). Conceptually, both the alliance and IPD are committed to uniting all participants based on the common goals, sharing risks and profits.

${ }^{*}$ Corresponding author. E-mail: guoshuwen@wust.edu.cn 
Research by the American Management Association has shown that collaboration can increase the profits for all supply chain partners by up to $3 \%$ (Khalfan \& McDermott, 2006). Long et al. (2009) stated that the dependence of participants on the alliance is an essential factor affecting the profit distribution. Meade and Lilesa (1997) proposed that scientific and fair profit distribution is the prerequisite for the normal operation and efficient development of the dynamic alliance, as well as a critical guarantee for the alliance members to achieve complementary resources and obtain profits. Profit distribution is a concern of all participants in the cooperative alliance and IPD and affects the satisfaction, enthusiasm of all parties and the success of the alliance and the IPD implementation. However, there is a lack of research on the profit distribution methods of the IPD mode in the AEC industry.

The objective of this study is to maximize the profit of an IPD team and to distribute the profits with efficiency and fairness. The main challenge is to determine the profitsharing model, which should be fully consistent with the agreement. A weight fuzzy cooperative game approach is used, and a quadratic programming model is established combining the feathers of the IPD. A case study is used to demonstrate the validity of the proposed approach. The results are expected to provide a reference for the profit distribution in IPD projects.

The remainder of this paper is arranged as follows. The first section is the literature review. Section 2 is devoted to preliminaries on cooperative games, fuzzy sets, $\alpha$-cut sets of triangular fuzzy numbers (TFNs), and interval-valued fuzzy numbers. The fuzzy profit distribution model based on alliance weights and its solution are introduced in Section 3. The model is used to ensure fair profit distribution to the IPD team. Furthermore, the quadratic programming model is modified after considering the efficiency of the fuzzy cooperative games. In Section 4, the proposed method is illustrated with a case study and is compared with other methods to demonstrate its validity, applicability, and superiority. The discussion is in Section 5 and conclusions at the end of the paper.

\section{Literature review}

In recent years, profit distribution has become a research hotspot in IPD related fields, such as supply chain and enterprise cooperation alliance. The same research concepts and methods have been used in these different fields. Quantitative research method of profit distribution is mainly to study the cooperative game situation of all parties by establishing mathematical model and analyzing the factors affecting profit distribution. Researchers have focused primarily on two perspectives: cooperative game theory and fuzzy mathematical theory.

Game theory provides a rigorous mathematical approach to evaluate and predict stakeholders' interactions. Cooperative game theory is used to analyze the situations in which a set of participants bargain to distribute the profit obtained from their collaboration. The resulting al- location is expressed as a payoff vector whose coordinates are the payoffs allocated to the participants. A game with a set of participants is modeled by a characteristic function that distributes the profit to each subset of participants' alliance so that the alliance members can obtain profit in the cooperate game. The cooperative game is a positive-sum game. During the game, the profit of one-party increases, whereas the profit of the other party is not threatened. Therefore, the overall profit will increase in the cooperative project. When multiple parties cooperate, the resulting profit exceeds the profits of each individual party. The solution of the cooperative game or the payoff vector is the distribution method of the cooperative alliance's profit. The solutions of cooperative games include the stable set, core, and distribution method. The most common solution of a cooperative game is the distribution method represented by the Shapley value.

Myerson (1997) analyzed the structure of cooperative games with the graph theory and used the Shapley value to assess the fair distribution and characteristic function of the profit of alliance distribution. Jia and Yokoyama (2003) discussed the profit distribution of an energy manufacturer alliance using the core and the nucleolus solution of the cooperative game. Teng et al. (2017) used the cooperative game method to analyze the profit distribution in the IPD project. They regarded the alliance in the IPD as a crisp alliance and adopted risk-sharing of the four stakeholders to modify the Shapley value for profit distribution. Pishdad-Bozorgi and Srivastava (2018) analyzed the relative effectiveness of risk and reward sharing arrangements in different IPD standard contracts on team cooperation motivation. Wang and Yuan (2019) solved the optimal solution set of a project's net income and improved overall satisfaction by combining a multi-objective profit distribution model with particle swarm optimization algorithm. Lin and Wang (2019) established a dynamic incentive model framework and applied the Hamilton-Jacobi-Bellman equation to solve Nash non-cooperative game using stochastic differential game theory. Liu and Cheng (2020) constructed that the asymmetric the Nash negotiation model to modify the measurement method of the factors affecting the income distribution to make it more consistent with the characteristics of dynamic alliances. Eissa et al. (2021) developed a conceptual framework using the Shapley value to address the marginal contribution of the participating parties not only for investment parties.

In addition to game theory, fuzzy mathematical theory is also commonly used to investigate profit distribution. After the fuzzy set theory was successfully applied to a controller system, it has been applied to other fields such as economics and management. Most of these applications use fuzzy numbers to describe the ambiguity and imprecision of data, which can be seen as an extension of real numbers. It is impossible to estimate the cost paid by the partners and the profits that should be obtained in the cooperation accurately due to non-determinacy factors, such as the level of effort and the degree of participation of the participants, the supervision mechanism, and the 
reward and punishment mechanism. This problem is an uncertainty problem and can be solved using fuzzy mathematics. The object of the fuzzy cooperative game is the maximization of profits of the limited rational players in a cooperative alliance and the method of efficient and fair profit distribution.

Alparslan-Gök et al. (2009) expanded the classical twoperson cooperative game theory and put forward the solution concepts and methods of the interval-valued two-person cooperative game theory. Li (2012) considered fuzzy matrix games in which the players' payoffs were expressed TFNs and players' pure and mixed strategies themselves were crisp. Han and Li (2016) used a continuous ordered weighted average operator and fuzzy cut-set method to convert an intuitionistic fuzzy alliance cooperative game with TFNs into a cooperative strategy of the intuitionistic fuzzy alliance at a confidence level and obtained the solutions of the cooperative games. Su and Yang (2018) introduced the confidence level of the $\lambda$-cut set and proposed a fuzzy average tree solution allocation model for payoff fuzzy graph cooperative games with interval characteristics. Ye et al. (2019) proposed a method for calculating the proportional residual distribution of TFNs in cooperative games according to the defined class alliance monotonicity condition. Su (2020) proposed a method of solving participants profit distribution cooperation countermeasures based on the TFNs in satisfying degree in entrepreneurial team. Abraham and Punniyamoorthy (2021) used three methods proposed to reduce the interval, i.e., the support of the fuzzy numbers, $\alpha$-cut sets, and the expected interval method to the precise interval.

These aforementioned studies proposed different methods of profit distribution and investigated the impact of profit distribution on the performance of a task or project, showing the importance of profit distribution to all participants in the project to achieve good results. These research results can be used in subsequent research on the profit distribution in the IPD projects. However, several limitations of the literature require consideration. First, some researchers directly implanted the profit distribution methods in the supply chains and enterprise cooperation alliances in the AEC industry. They did not fully consider the characteristics of the AEC industry, weakening the impact of the heterogeneity of the construction enterprise on the profit distribution in a project. The completion of a project, the owner, the architect, the builder, and the supplier, and so on are indispensable. They form a grand alliance to participate in profit distribution. At the same time, studies on project profit distribution in IPD projects typically considered only the fairness of profit distribution but not its efficiency. Thus, the profit distribution did not reach the Pareto optimal. In addition, most researchers analyzed crisp cooperative games and rarely used fuzzy values to evaluation profit distribution in the IPD projects. In practice, the level of effort, the degree of participation, and the supervision mechanism of each participant result in uncertainty in profit distribution. Moreover, few researchers used interval-valued fuzzy numbers to analyze profit distribution in IPD.
Although the practical effect of the profit distribution in IPD is highly significant, there are relatively few theoretical and empirical research studies on this topic. In particular, few studies used fuzzy value considering the efficiency of each player on the profit distribution in IPD projects. The success of the IPD model is inseparable from a fair profit distribution mechanism. IPD emphasizes the efficient cooperation of the alliance members, where each participant is independent with their own interests. The consideration of the alliance weights and fair profit distribution is part of the smooth implementation of IPD. This approach can provide a new perspective, enrich the theoretical research of profit distribution in IPD projects, and promote IPD and efficient, fair, and healthy development of the AEC industry. IPD has the advantages of resource aggregation and cooperation among construction project participants. Thus, it is bound to be more widely used in the future.

This paper aims to provide a framework to analyze profit distribution in IPD projects. Interval-valued fuzzy numbers are introduced to investigate the profit distribution in IPD project using a cooperative game. We propose a method based on a quadratic programming model of interval-valued fuzzy numbers. The method considers the importance of the alliance and the efficiency of participants to distribute profits in IPD projects. The results of the proposed method are compared with those of crisp cooperative games using a case study. This research provides a new effective fuzzy cooperative game method and can be extended to provide a solution for IPD profit distribution.

\section{Preliminaries}

A cooperative game is devoted to solve the problem of the cooperation among multiple players and achieve a reasonable distribution of jointly obtained profits. It establishes a mechanism of mutual trust and commitment through agreements, negotiations, and information exchanges to achieve the Pareto optimum of the union. The cooperative game has the following four key features: 1) Common interests. These are the prerequisite for cooperation and alliance; 2) Information exchange. The cooperative game emphasizes the smooth exchange of information between participants; 3) Voluntariness, equality, and mutual profit; 4) A mandatory contract. The contract resulting from negotiation in the cooperative game is legally binding.

In IPD projects, all participants voluntarily and equally ally with a common goal and share common interests in the cooperative game. They can use the information-sharing platform provided by BIM and other technologies to communicate smoothly and share risks/rewards. The core players are exempted from responsibility and share profits. There is a highly trusted cooperative relationship. At the start of an IPD project, the owner and the core team must agree on a legal structure for the core team, such as multiple independent contracts, a single multi-party contract, a joint venture, or a limited liability company (CMAA, 2010). In this mode, a reasonable profit distribution is not 
only the key condition of the smooth implementation but also an important condition to measure the success of the project. The profit distribution strategy among enterprises in IPD projects is the result of rational choice in the cooperation and competition among players. In summary, the alliance of project participants in IPD projects is a typical cooperative game problem.

The profit distribution is manifested in the continuation of the production or exchange in practical activities. It is an economic operation in which social products are divided among different stakeholders. During project implementation, several independent economic entities form a joint entity. Therefore, all participants in the alliance are the subject of profit distribution. A Cooperative game can be regarded as a complete set of cooperative behaviors, which not only achieve the basic income goals of all parties, but also produce some cooperative "surplus". As a consequence, the concept of a cooperative game is the reasonable distribution of this "surplus" and the apportioning of the cost of cooperation.

The profit distribution system consists of a cost compensation and an incentive bonus. Cost compensation is the remuneration for the services or labor provided by the IPD stakeholders through negotiation, including direct and indirect costs. The incentive bonus distribution is associated with the IPD goals, including target achievement awards and incentive awards, which are the core content of the profit distribution system. The focus of profit distribution in this study is the incentive bonus.

It first introduces some preliminaries of cooperative games, such as TFNs, A-cut sets, and interval-valued fuzzy numbers, and then establishes a cooperative game pre-allocation model that considers fairness and alliance weight, which is solved by the least-squares method in quadratic programming to get the preliminary profit distribution. On this basis, a modified quadratic programming model is established by considering the validity constraints. The Lagrange multiplier method is used to solve the model to obtain the revised profit distribution.

\subsection{Crisp cooperative game}

In the cooperation of the members of the enterprise alliance, the set of all participating enterprises is $N=\{1,2$, ..., $n\}$. A crisp alliance $X$ is a subset of $N$, and the class of all crisp alliances of $X$ is denoted by $P(X)$ (Shapley, 1953). $X$ and $Y$ are disjoint crisp alliances. The $X$ 's payoff value $v: P(X) \rightarrow \mathbb{R}^{+}$satisfies:

$$
\begin{aligned}
& v(\varnothing)=0 ; \\
& v(X \cup Y) \geq v(X)+v(Y), \forall X, Y \in P(X), X \cap Y=\varnothing,
\end{aligned}
$$

where $\mathbb{R}^{+}$is the set of positive real numbers. $G_{0}(N)$ denotes the crisp superadditive cooperative games. If a crisp cooperative game $(N, v)$ satisfies the above two conditions, we define it as a convex cooperative game. Condition (2) indicates that the expected return (the payoff function) $v$ of the cooperative game alliance formed by the enter- prise is a monotonous convex function with superadditivity. The profit distribution of the enterprise alliance is obtained by solving the cooperative game $(N, v)$.

An imputation for a crisp cooperative game $v \in G_{0}$ (N) is a vector $X=\left(x_{1}, \ldots, x_{n}\right)$ satisfying: (i) $\sum_{i=1}^{n} x_{i}=v(N)$, (ii) $x_{i} \geq v(\{i\}), i=1,2, \ldots, n$. The condition (i) is termed the efficiency property, and (ii) is called the individual rationality property (Yu \& Zhang, 2019). Thus, the imputation is a vector describing the efficiency property and individual rationality. Let $I(N, v)$ represent the sets of all the imputation of the crisp game $v \in G_{0}(N)$, and $X(N, v)$ denote the set of solutions verifying the efficiency property, i. e.,

$$
\begin{aligned}
& I(N, v)=\left\{x \in \mathbb{R}^{+} \mid \sum_{i=N} x_{i}=v(N), x_{i} \geq v(\{i\}), \forall i \in N\right\}, \\
& X(N, v)=\left\{x \in \mathbb{R}^{+} \mid \sum_{i=N} x_{i}=v(N)\right\} .
\end{aligned}
$$

\subsection{Triangular fuzzy numbers and $\alpha$-cut sets}

In this section, the basic definitions of fuzzy set theory are reviewed. In most applications fuzzy numbers describe the fuzziness and imprecision of data. They can be regarded as a generalization of real numbers.

Let $X$ denote a universal set. The fuzzy subset $\tilde{M}$ of $X$ is defined by its membership function $\mu_{\tilde{M}}: X \rightarrow[0,1]$ which assigns a real number $\mu_{\tilde{M}}(X)$ in the interval $[0$, 1] to each element $x \in X$, where the value of $\mu_{\tilde{M}}(X)$ at $x$ shows the degree of membership of $x$ in $\tilde{M}$ (Dubois \& Prade, 1980).

For any $\alpha \in[0,1]$, we define the $\alpha$ cut-set of $\tilde{M}$ as $\tilde{M}_{\alpha}=\left\{x \mid \mu_{\tilde{M}}(x) \geqslant \alpha, x \in X\right\}$, where $\alpha$ is the confidence level or confidence. If $\tilde{M}$ is a normal fuzzy set, there exists $x \in X$ so that $\mu_{\tilde{M}}(x)=1$. In the theory and application of fuzzy sets, it is often assumed that all fuzzy sets meet the requirements of regularity. Otherwise, they can be simply transformed. Therefore, the fuzzy sets discussed in this study are assumed to be regular fuzzy sets. For any $\alpha \in[0,1]$, the $\alpha$ cut-set of $\tilde{M}_{\alpha}$ is a bounded closed interval on the real number $\mathbb{R}^{+}$, which is denoted as $\tilde{M}_{\alpha}=\left[a_{\alpha}^{l}(x), a_{\alpha}^{r}(x)\right]$.

We denote $\Gamma(M)$ as the entire fuzzy set on the real number $\mathbb{R}^{+} \rightarrow[0,1]$. Let $\tilde{M}$ be a TFN, and its membership function is denoted as $\mu_{\tilde{M}}(x)$. The triangular fuzzy membership function of $\tilde{M}$ can be expressed as:

$$
\mu_{\tilde{M}}(x)= \begin{cases}\left(x-a^{l}\right) /\left(a^{m}-a^{l}\right) & \text { if } a^{l} \leq x<a^{m} \\ 1 & \text { if } x=a^{m} \\ \left(a-x^{r}\right) /\left(a^{r}-a^{m}\right) & \text { if } a^{m}<x \leq a^{r} \\ 0 & \text { else }\end{cases}
$$

where $a^{l} \leq a^{m} \leq a^{r}$. The membership function is triangular, and the TFN is often abbreviated as $\tilde{a}=\left(a^{l}, a^{m}, a^{r}\right) \cdot a^{l}$ and $a^{r}$ represent the lower and upper limits of the fuzzy number $\tilde{M}$ respectively. $a^{m}$ is the mean of $\tilde{M}$, which is the most probable value. When $a^{l}=a^{m}=a^{r}$, the TFN $\tilde{a}=$ $\left(a^{l}, a^{m}, a^{r}\right)$ degenerates into a real number. A real number is a special form of a TFN. 
Arithmetical operations and the $\alpha$-cuts of the TFNs are used. Let $\tilde{a}=\left(a^{l}, a^{m}, a^{r}\right)$ and $\tilde{b}=\left(b^{l}, b^{m}, b^{r}\right)$ be two arbitrary non-negative TFNs; then

$$
\begin{aligned}
& \tilde{a} \oplus \tilde{b}=\left(a^{l}+b^{l}, a^{m}+b^{m}, a^{r}+b^{r}\right) \\
& \lambda \tilde{a}=\left\{\begin{array}{l}
\left(\lambda a^{l}, \lambda a^{m}, \lambda a^{r}\right) \text { if } \lambda \geq 0 \\
\left(\lambda a^{r}, \lambda a^{m}, \lambda a^{l}\right) \text { if } \lambda<0
\end{array} .\right.
\end{aligned}
$$

and

Equations (5) and (6) indicate that the sum of the arbitrary non-negative TFNs and the product of an arbitrary real number and an arbitrary non-negative TFN are TFNs.

It can be derived from Eqn (4) that $a^{L}(\alpha)=$ $\alpha a^{m}+(1-a) \alpha^{l}$ and $a^{R}(\alpha)=\alpha a^{m}+(1-a) \alpha^{R}$. In particular, $\tilde{a}(1)=\left\{x \mid \mu_{\tilde{M}}(x) \geqslant 1\right\}=\left[a^{L}(1), a^{R}(1)\right]=\left[a^{m}, a^{m}\right]=a^{m}$ and $\tilde{a}(0)=\left\{x \mid \mu_{\tilde{M}}(x) \geqslant 0\right\}=\left[a^{L}(0), a^{R}(0)\right]=\left[a^{l}, a^{r}\right]$.

According to the operations over intervals (Zhao \& Liu, 2018), it follows that:

$$
\begin{aligned}
& {\left[a^{L}(\alpha), a^{R}(\alpha)\right]=\alpha\left[a^{m}, a^{m}\right]+[1-\alpha]\left[a^{l}, a^{r}\right]=} \\
& \alpha \tilde{a}(1)+[1-\alpha] \tilde{a}(0),
\end{aligned}
$$

which means that any $\alpha$-cut set of a TFN can be directly obtained from its 1-cut and 0-cut, as depicted in Figure 1.

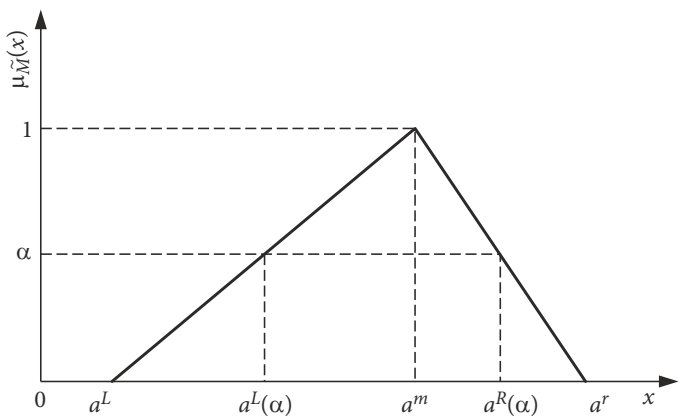

Figure 1. Any $\alpha$-cut set of a TFN $\tilde{a}=\left(a^{l}, a^{m}, a^{r}\right)$

\subsection{Interval-valued fuzzy numbers}

The fuzzy cooperative game is represented by an ordered binary group $(N, \tilde{v})$, where $\{N=1,2, \ldots, n\}$ represents the set of all participants, and ${ }^{\tilde{v}}$ is the characteristic value (or characteristic function) of the fuzzy alliance denoted by TFNs. $\tilde{v}(S)$ is a TFN for any alliance $S \subseteq N$. Consequently, $\tilde{v}(S)=\left(a_{S}^{l}, a_{S}^{m}, a_{S}^{r}\right)$, where $a_{S}^{l} \leq a_{S}^{m} \leq a_{S}^{r}$ and $a_{S}^{l}>0$. Moreover, we use a quadratic programming model and solve the cooperative games with characteristic values expressed by the TFNs based on the concept of the $\alpha$-cut sets.

As stated before, for any $\alpha \in[0,1]$, the $\alpha$-cut sets of the payoffs $\tilde{v}(S)=\left(a_{S}^{l}, a_{S}^{m}, a_{S}^{r}\right)$ can be expressed as $\tilde{v}(S)(\alpha)=$ $\left[\alpha a_{S}^{m}+(1-\alpha) a_{S}^{l}, \alpha a_{S}^{m}+(1-\alpha) a_{S}^{r}\right]$ (Babbar et al., 2013). For simplicity, $\alpha a_{S}^{m}+(1-\alpha) a_{S}^{l}$ and $\alpha a_{S}^{m}+(1-\alpha) a_{S}^{r}$ are abbreviated as $v^{L}(S)$ and $v^{R}(S)$ respectively, i.e., $\tilde{v}(S)$ $(\alpha)=\left[v^{L}(S), v^{R}(S)\right]$. For the TFN-type alliance values in the fuzzy cooperative games, each player has a TFN-type distribution $x_{i}(\alpha)=\left(x_{i}^{l}, x_{i}^{m}, x_{i}^{r}\right), i \in N$, in the grant alliance. $x_{i}(\alpha)$ use a TFN-typed $\alpha$-cut sets to express $x_{i}(\alpha)=$ $\left[x_{i}^{L}(\alpha), x_{i}^{R}(\alpha)\right]=\left[\alpha x_{i}^{m}+(1-\alpha) x_{i}^{l}, \alpha x_{i}^{m}+(1-\alpha) x_{i}^{r}\right]$.
Let $x(S)(\alpha)=\sum_{i \in S} x_{i}(\alpha)$, which denotes the TFN-type $\alpha$-cut set of all players in the alliance. We use an interval value representation, $x(S)(\alpha)=\left[\sum_{i \in S} x_{i}^{L}(\alpha), \sum_{i \in S} x_{i}^{R}(\alpha)\right]$.

\section{Profit distribution in the fuzzy cooperative game alliance}

\subsection{Cooperative game pre distribution model considering alliance weight}

Methods for solving the fuzzy cooperative games, whose payoff value is represented as interval-valued fuzzy numbers, do not consider the alliance weights. This study considers the alliance weights to reflect the influence of the importance of different alliances on the allocation scheme. We employ interval-valued fuzzy numbers to represent the alliance and solve the model. This approach is called the weight fuzzy distribution model, which is defined in Eqn (8). It is solved by determining the distribution of the interval-valued fuzzy numbers for each player participating in the alliance.

We denote $|S|$ as the number of players in the alliance. $\omega(s)$ is the weight of the alliance $S \subseteq N$, which $\omega(s)$ is a real number. In practical situations, the more players an alliance has, the more influence the alliance has. Thus, we assume that $\omega(s)$ is determined by the number of players in the alliance. Specifically, if $|S|=n$, then the weight of this alliance is $n$ times the weight of the alliance of $|S|=1$. Thus, in a cooperative game composed of $n$ players, the weight of $|S|=i,(i=1,2, \ldots, n)$ is $\omega(s)=\frac{i}{C_{n}^{1}+2 C_{n}^{2}+3 C_{n}^{3}+\cdots+n C_{n}^{n}}$, and $\sum_{S \subseteq N} \omega(s)=1$.

We use the least squares method to solve the convex function optimization problem because the Euclidean distance is easy to calculate, and the characteristic properties remain unchanged after the transformation of different representation domains. The similarity between the two fuzzy numbers ${ }^{\tilde{v}}(S)(\alpha)$ and $x(S)(\alpha)$ considering the weight of the alliance is measured by the Euclidean distance.

$$
\begin{aligned}
& D(x(S)(\alpha), \tilde{v}(S)(\alpha))=\left(\sum _ { S \subseteq N } \omega ( S ) \left(\sum _ { i \in S } \left(x_{i}^{L}(\alpha)-\right.\right.\right. \\
& \left.v^{L}(S)(\alpha)\right)^{2}-\left(\sum_{i \in S}\left(x_{i}^{R}(\alpha)-v^{R}(S)(\alpha)\right)^{2}\right)^{1 / 2} .
\end{aligned}
$$

The squared loss function is:

$$
\begin{aligned}
& L(x(\alpha))=\sum_{S \subseteq N}(D(x(S)(\alpha), \tilde{v}(S)(\alpha)))^{2}= \\
& \sum_{S \subseteq N} \omega(S)\left(\sum_{i \in S}\left(x_{i}^{L}(\alpha)-v^{L}(S)(\alpha)\right)^{2}-\right. \\
& \left(\sum_{i \in S}\left(x_{i}^{R}(\alpha)-v^{R}(S)(\alpha)\right)^{2},\right.
\end{aligned}
$$

where $x(\alpha)=\left(x_{1}(\alpha), x_{2}(\alpha), \ldots, x_{n}(\alpha)\right)^{T}$ is the vector of the $\alpha$-cut set of the TFN-typed imputations for all players in the grand alliance $N . L(x(a))$ is a type of squared loss 
functions. The squared loss is the sum of the squares of the difference between the prediction and the label, i.e., the sum of the squared residuals. Our goal is to minimize this objective function. The profit distribution of all participants in the optimized $a$-cut set can be expressed as follows:

$$
\begin{aligned}
& \min \left\{L(x(\alpha))=\sum_{S \subseteq N} \omega(S)\left[\left(\sum_{i \in S} x_{i}^{L}(\alpha)-\right.\right.\right. \\
& \left.\left.v^{L}(S)(\alpha)\right)^{2}+\left(\sum_{i \in S} x_{i}^{R}(\alpha)-v^{R}(S)(\alpha)\right)^{2}\right] .
\end{aligned}
$$

We take the partial derivative of Eqn (9) and set it to 0 to obtain the regular equation:

$\left\{\begin{array}{l}\sum_{S \subseteq N: j \in S} \omega(S) \sum_{i \in S} x_{i}^{L}(\alpha)=\sum_{S \subseteq N: j \in S} \omega(S) v^{L}(S)(\alpha) \quad(j=1,2, \cdots, n) \\ \sum_{S \subseteq N: j \in S} \omega(S) \sum_{i \in S} x_{i}^{R}(\alpha)=\sum_{S \subseteq N: j \in S} \omega(S) v^{R}(S)(\alpha) \quad(j=1,2, \cdots, n)\end{array}\right.$.

We expand the above Eqn (10) respectively to obtain the following two equations:

$\left\{\begin{array}{c}a_{11} x_{1}^{L}(\alpha)+a_{12} x_{2}^{L}(\alpha)+a_{13} x_{3}^{L}(\alpha)+\cdots+a_{1 n} x_{n}^{L}(\alpha)=\sum_{S \subseteq N: 1 \in S} \omega(S) v^{L}(S)(\alpha) \\ a_{21} x_{1}^{L}(\alpha)+a_{22} x_{2}^{L}(\alpha)+a_{23} x_{3}^{L}(\alpha)+\cdots+a_{2 n} x_{n}^{L}(\alpha)=\sum_{S \subseteq N: 2 \ell S} \omega(S) v^{L}(S)(\alpha) \\ \cdots \\ a_{n 1} x_{1}^{L}(\alpha)+a_{n 2} x_{2}^{L}(\alpha)+a_{n 3} x_{3}^{L}(\alpha)+\cdots+a_{n n} x_{n}^{L}(\alpha)=\sum_{S \subseteq N: n \in S} \omega(S) v^{L}(S)(\alpha)\end{array}\right.$
$\left\{\begin{array}{c}a_{11} x_{1}^{R}(\alpha)+a_{12} x_{2}^{R}(\alpha)+a_{13} x_{3}^{R}(\alpha)+\cdots+a_{1 n} x_{n}^{R}(\alpha)=\sum_{S \subseteq N: 1 \in S} \omega(S) v^{R}(S)(\alpha) \\ a_{21} x_{1}^{R}(\alpha)+a_{22} x_{2}^{R}(\alpha)+a_{23} x_{3}^{R}(\alpha)+\cdots+a_{2 n} x_{n}^{R}(\alpha)=\sum_{S \subseteq N: 2 \in S} \omega(S) v^{R}(S)(\alpha) \\ \cdots \\ a_{n 1} x_{1}^{R}(\alpha)+a_{n 2} x_{2}^{R}(\alpha)+a_{n 3} x_{3}^{R}(\alpha)+\cdots+a_{n n} x_{n}^{R}(\alpha)=\sum_{S \subseteq N: n \in S} \omega(S) v^{R}(S)(\alpha)\end{array}\right.$

According to the permutation and combination theory, we can calculate the following formula:

(1) When $i=j(i, j \in\{1,2, \ldots n\})$,

$$
\begin{aligned}
& a_{i j}=\omega(1) C_{n-1}^{0}+\omega(2) C_{n-1}^{1}+\omega(3) C_{n-1}^{2}+\cdots+ \\
& \omega(n-1) C_{n-1}^{n-2}+\omega(n) C_{n-1}^{n-1} ;
\end{aligned}
$$

(2) When $i \neq j(i, j \in\{1,2, \ldots n\})$,

$$
\begin{aligned}
& a_{i j}=\omega(1) C_{n-1}^{0}+\omega(2) C_{n-1}^{1}+\omega(3) C_{n-1}^{2}+\cdots+ \\
& \omega(n-1) C_{n-1}^{n-3}+\omega(n) C_{n-1}^{n-2} .
\end{aligned}
$$

Denote $a=\omega(1) C_{n-1}^{0}+\omega(2) C_{n-1}^{1}+\omega(3) C_{n-1}^{2}+\cdots+$

$\omega(n-1) C_{n-1}^{n-2}+\omega(n) C_{n-1}^{n-1}$,

$b=\omega(1) C_{n-1}^{0}+\omega(2) C_{n-1}^{1}+\omega(3) C_{n-1}^{2}+\cdots+$

$\omega(n-1) C_{n-1}^{n-3}+\omega(n) C_{n-1}^{n-2}$.

Then, $a_{i j}=\left\{\begin{array}{ll}a, & (i=j ; i, j \in\{1,2, \cdots, n\}) \\ b, & (i=j ; i, j \in\{1,2, \cdots, n\})\end{array}\right.$.

Denote $x^{L}(\alpha)=\left(x_{1}^{L}(\alpha), x_{2}^{L}(\alpha) \cdots x_{n}^{L}(\alpha)\right)^{T}$,

$x^{R}(\alpha)=\left(x_{1}^{R}(\alpha), x_{2}^{R}(\alpha) \cdots x_{n}^{R}(\alpha)\right)^{T}$,

$B^{L}(\alpha)=\left(\sum_{S \subseteq N: 1 \in S} v^{L}(S)(\alpha), \sum_{S \subseteq N: 2 \in S} v^{L}(S)(\alpha), \cdots\right.$,

$\left.\sum_{S \subseteq N: n \in S} v^{L}(S)(\alpha)\right)^{T}$,

$$
\begin{aligned}
& B^{R}(\alpha)=\left(\sum_{S \subseteq N: 1 \in S} v^{R}(S)(\alpha), \sum_{S \subseteq N: 2 \in S} v^{R}(S)(\alpha), \cdots,\right. \\
& \left.\sum_{S \subseteq N: n \in S} v^{R}(S)(\alpha)\right)^{T}
\end{aligned}
$$

and $A=\left(a_{i j}\right)_{n \times n}=\left(\begin{array}{cccc}a & b & \cdots & b \\ b & a & \cdots & b \\ \cdots & \cdots & \cdots & \vdots \\ b & b & \cdots & a\end{array}\right)_{n \times n}$.

Thus, Eqn (10) can be expressed in the following matrix format:

$$
\left\{\begin{array}{l}
A x^{L}(\alpha)=B^{L}(\alpha) \\
A x^{R}(\alpha)=B^{R}(\alpha)
\end{array} .\right.
$$

According to the matrix multiplication algorithm, the lower limit of the optimal distribution vector of the players in the alliance can be solved using Eqn (11). The upper limit is determined using Eqn (12):

$$
\left\{\begin{array}{l}
x^{L}(\alpha)=A^{-1} B^{L}(\alpha) \\
x^{R}(\alpha)=A^{-1} B^{R}(\alpha)
\end{array}\right. \text {. }
$$

Therefore, we can obtain the profit distribution of all players in the interval-valued fuzzy numbers, which can be expressed as $x_{i}(\alpha)=\left[x_{i}^{L}(\alpha), x_{i}^{R}(\alpha)\right](i=1,2, \ldots, n)$. Then, we can calculate $x_{i}(0)$ and $x_{i}(1)$. According to the fuzzy sets' representation theorem $(\mathrm{Li}, 2012)$. The interval-valued fuzzy numbers imputation of player $i \in N$ can be denoted by $x_{i}=\left(x_{i}^{l}, x_{i}^{m}, x_{i}^{r}\right)=\left(x_{i}^{L}(0), x_{i}^{L}(1), x_{i}^{R}(0)\right)=$ $\left(x_{i}^{L}(0), x_{i}^{R}(1), x_{i}^{R}(0)\right)$, where $x_{i}^{L}(1)=x_{i}^{R}(1)$.

\subsection{Redistribution considering alliance weights and effectiveness}

In Section 3.1, a fuzzy profit distribution model was established, and the quadratic programming model was solved to obtain the profit distribution of the cooperative game, where the payoff value is expressed as an interval-valued fuzzy number. This approach ensures that the profit distribution is fair.

The concept of solving distribution problems needs to secure the fairness of its solution, because the solution concept can only be successfully applied in practice if all participants accept the solution as fair. The profits of a cooperation must be distributed completely. This entails that the profits cannot be preserved and distributed in the future, nor can there be more distributed than the cooperation has collectively obtained. Consequently, the sum of all payoffs must equal the collectively earned profits. This is called efficiency (Jene \& Zelewski, 2014).

In the following, we modify the above model by considering the efficiency of the cooperative game so that the sum of the profits obtained by the players participating in the cooperative alliance is equal to the profit of the grand alliance (Zhao \& Liu, 2018). We combine Eqns (3) and (9) to obtain the modified equation:

$$
\min \left\{L(x(\alpha))=\sum_{S \subseteq N} \omega(S)\left[\left(\sum_{i \in S} x_{i}^{L}(\alpha)-v^{L}(S)(\alpha)\right)^{2}+\right.\right.
$$




$$
\begin{aligned}
& \left.\left.\left(\sum_{i \in S} x_{i}^{R}(\alpha)-v^{R}(S)(\alpha)\right)^{2}\right]\right\} \\
& \text { s.t. }\left\{\begin{array}{l}
\sum_{i=1}^{n} x_{i}^{L}(\alpha)=v^{L}(N)(\alpha) \\
\sum_{i=1}^{n} x_{i}^{R}(\alpha)=v^{R}(N)(\alpha)
\end{array}\right.
\end{aligned}
$$

We create the Lagrange function by using the Lagrange multiplier method as follows:

$$
\begin{aligned}
& L(x(\alpha), \lambda(\alpha), \mu(\alpha))= \\
& \sum_{S \subseteq N} \omega(S)\left[\left(\sum_{i \in S} x_{i}^{L}(\alpha)-v^{L}(\alpha)\right)^{2}+\right. \\
& \left.\left(\sum_{i \in S} x_{i}^{R}(\alpha)-v^{R}(\alpha)\right)^{2}\right)+ \\
& \lambda(\alpha)\left(\sum_{i \in S} x_{i}^{L}(\alpha)-v^{L}(\alpha)\right)+ \\
& \mu(\alpha)\left(\sum_{i \in S} x_{i}^{R}(\alpha)-v^{R}(\alpha)\right) ; \\
& L(x(\alpha), \lambda(\alpha), \mu(\alpha))= \\
& \sum_{S \subseteq N} \omega(S)\left[\left(\sum_{i \in S} x_{i}^{L}(\alpha)-v^{L}(\alpha)\right)^{2}+\right. \\
& \left.\left(\sum_{i \in S} x_{i}^{R}(\alpha)-v^{R}(\alpha)\right)^{2}\right]+ \\
& \lambda(\alpha)\left(\sum_{i \in S} x_{i}^{L}(\alpha)-v^{L}(\alpha)\right)+ \\
& \mu(\alpha)\left(\sum_{i \in S} x_{i}^{R}(\alpha)-v^{R}(\alpha)\right) .
\end{aligned}
$$

Then, the $\alpha$-cut sets of the allocations of all participants, which represents the solution of the fuzzy cooperative game $v$ with TFN-typed alliance values are the solution of the quadratic programming model as follows:

$$
\begin{aligned}
& \min \{L(x(\alpha), \lambda(\alpha), \mu(\alpha))= \\
& \sum_{S \subseteq N} \omega(S)\left[\left(\sum_{i \in S} x_{i}^{L}(\alpha)-v^{L}(\alpha)\right)^{2}+\right. \\
& \left.\left(\sum_{i \in S} x_{i}^{R}(\alpha)-v^{R}(\alpha)\right)^{2}\right]+ \\
& \lambda(\alpha)\left(\sum_{i \in S} x_{i}^{L}(\alpha)-v^{L}(\alpha)\right)+ \\
& \left.\mu(\alpha)\left(\sum_{i \in S} x_{i}^{R}(\alpha)-v^{R}(\alpha)\right)\right\} .
\end{aligned}
$$

Solving Eqn (14) is similar to solving Eqn (9). We take the partial derivatives of $L(x(\alpha), \lambda(\alpha), \mu(\alpha))$ in Eq. (14) with regard to variables $x_{i}^{L}(\alpha), x_{i}^{R}(\alpha), \lambda(\alpha)$, and $\mu(\alpha)$, respectively and set them as 0 . Subsequently, the equations are transformed into a block matrix to obtain the coefficient matrix of the equations. Further, we obtain the solution of Eqn (14) as follows:

$$
\left\{\begin{array}{l}
x^{L^{*}}(\alpha)=x^{L}(\alpha)+\frac{1}{n}\left(v^{L}(N)(\alpha)-\sum_{i=1}^{n} x_{i}^{L}(\alpha)\right) e \\
x^{R^{*}}(\alpha)=x^{R}(\alpha)+\frac{1}{n}\left(v^{R}(N)(\alpha)-\sum_{i=1}^{n} x_{i}^{R}(\alpha)\right) e
\end{array},\right.
$$

where $x^{L^{*}}(\alpha)=\left(x_{1}^{L^{*}}(\alpha), x_{2}^{L^{*}}(\alpha), \ldots x_{n}^{L^{*}}(\alpha)\right)^{T}$, $x^{R^{*}}(\alpha)=\left(x_{1}^{R^{*}}(\alpha), x_{2}^{R^{*}}(\alpha), \ldots x_{n}^{R^{*}}(\alpha)\right)^{T}$.

This analysis indicates that if the effectiveness of the fuzzy alliance is considered, the payoff distribution of all players expressed by the $\alpha$-cut sets can be rewritten as $x_{i}^{*}(\alpha)=\left[x_{i}^{L^{*}}(\alpha), x_{i}^{R^{*}}(\alpha)\right](i=1,2, \ldots, n)$, which $x_{i}^{L^{*}}(\alpha)$ and $x_{i}^{R^{*}}(\alpha)$ can be solved by Eqn (15), respectively. As described in Section 3.2, we use the representation theorem of the fuzzy sets (Moore, 1979) so that the TFN-type imputation of the player $i \in N$ can be expressed as $x_{i}^{*}=\left(x_{i}^{l^{*}}, x_{i}^{m^{*}}, x_{i}^{r^{*}}\right)=\left(x_{i}^{L^{*}}(0), x_{i}^{L^{*}}(1), x_{i}^{R^{*}}(0)\right)=$ $\left(x_{i}^{L^{*}}(0), x_{i}^{R^{*}}(1), x_{i}^{R^{*}}(0)\right)$.

\subsection{Criteria for a successful practical application}

The purpose of this section is to examine the criteria conditions for the successful practice of the method presented above in IPD project. The following criteria are conditions for successful practical application of the cooperative game profit distribution:

(1) Efficiency. The sum of all payoffs must equal the collectively obtained profits. This has been explained in Section 3.2 above.

(2) Individual rationality. The payment obtained by each player in the cooperative game should not be lower than the payoff he gets if he works alone.

(3) Transparency. The profit distribution scheme in IPD projects must be easily communicable and intelligible to implement for application smoothly. Therefore, the method must be sufficiently transparent.

(4) Information sharing. Each collaborative solution has different information requirements. If some information is not disclosed by some participants, it is difficult to ensure the fairness and accuracy of the distribution scheme.

The above four points are consistent with the characteristics of IPD projects. Therefore, the model in this paper has strong applicability.

\section{Results}

\subsection{An example case}

The case data were obtained from the case sets in IPD projects summarized by the American Institute of Architects [AIA] (2010). As a successful application of IPD, the St. Clare Health Centre is a $430,000 \mathrm{ft}^{2}$ replacement hospital located in Fenton, Missouri, USA. The health center has a tower composed of six floors and 154 beds, with an $85000 \mathrm{ft}^{2}$ medical office building and a $75000 \mathrm{ft}^{2}$ outpatient nursing center. The owner (SSM Healthcare), the architect (HGA Architects and Engineers), and the builder (Alberici Constructors) entered into a tripartite IPD contract using the integrated form of agreement (IFOA) based on collaboration and lean methodologies. The architect and builder combined their contingencies and are jointly responsible for construction errors and design omissions. 
"Lean partners", i.e., subcontractors within the shared risk/ reward circle, included mechanical, electrical, and plumbing (MEP), wall and ceiling framing and finish, and fire protection. Smaller pieces of the work were bid out traditionally. According to this agreement, each party must treat the other partners equally. We use this case to verify the proposed profit distribution model.

\subsection{Calculation of distributable profits of the alliance}

In this case, the core players composed of the owner, the architect, and the builder are respectively denoted as player 1, player 2, and player 3 . They form a cooperative alliance with three players, and the cooperative game model is $\{N, v\} . v(s)$ is the total payoff of the alliance. In the exante allocation scheme, it is assumed that the future profit is an uncertain random variable and the future costs are also the expected cost.

In the traditional delivery mode, such as DBB, DB, all participants fulfill their contractual obligations and perform the project operation using normal procedures when participating in the project, and obtain the average profit in the industry. Therefore, in the traditional delivery mode, the additional profit allocated to each player is 0 , that is $v(\{1\})=v(\{2\})=v(\{3\})=0$.

In the AIA case, we calculate the real number of the expected cost of each project player in this IPD project. Since the average profit rate of each industry is a statistical value, and the profits of the three participants are uncertain due to the level of effort and the degree of participation of each participant and the influence of other reward and punishment mechanisms, the expected profit value can be expressed by interval-valued fuzzy numbers. We assume that the accurate expected return calculated from the average profit rate of the industry fluctuates by $5 \%$. The average profit rate and expected return of the three players in the IPD project are listed in Table 1.

All participants in the alliance cooperate closely, exchange information smoothly, share resources, and increase their efforts as much as possible to achieve high team performance and add value to the alliance and value chain. For example, the owner and the architect cooperate initially, communicating and strengthening the cooperation in all aspects. This approach optimizes the design scheme, shortens the design time, strengthens the management of milestones effectively, reduces the cost of the owner and the whole life cycle cost of the project, and improves owner satisfaction.
Through BIM and other information communication platforms and lean methodologies, all participants communicate effectively, minimize design changes and rework, reduce waste, reduce cost, ensure quality, speed up progress, ensure adherence to the construction period, improve work efficiency, and achieve safe and effective production.

IPD results in greater efficiencies. The United Kingdom's Office of Government Commerce [UKOGC] estimated that construction cost savings of up to $30 \%$ in the cost of construction could be achieved where integrated teams promote continuous improvement over a series of construction projects. The UKOGC further estimated that single projects employing integrated supply teams could achieve savings of $2-10 \%$ in the cost of construction (UKOGC, 2007). In a case study of IPD conducted by Autodesk Inc., AEC Solutions Division Headquarters, the contract established an Incentive Compensation Layer (ICL) in which the architects' and builders' anticipated profit was put at risk (AIA, 2010). If the profits were exceeded in measurable ways, the firms were eligible for additional compensation. The ICL could be adjusted from minus $20 \%$ to plus $20 \%$ depending on whether the project goals were met or exceeded.

Therefore, the expected return of single members of a multi-member alliance is added to increase the percentage. The floating ratio does not affect the distribution rate of each member participating in the alliance but only affects the distribution value of each player at the same time. Moreover, it is assumed that a bi-member alliance will increase the profit by $10 \%$, and a tri-member alliance will increase the profit by $20 \%$. Thus, the expected profit for multiple members can be obtained, as listed in Table 2.

Table 2. Distributable profits of the alliance in the fuzzy cooperative game (unit: thousand dollars)

\begin{tabular}{|l|c|c|}
\hline Alliance & $\begin{array}{c}\text { Expected earnings of } \\
\text { alliance }\end{array}$ & $\begin{array}{c}\text { Alliance distributable } \\
\text { profit }\end{array}$ \\
\hline$\hat{v}(\{1,2\})$ & $(17464.8,18539.7$, & $(1601.0,1685.3$, \\
$19466.7)$ & $1769.5)$ \\
\hline$\hat{v}(\{1,3\})$ & $(27828.4,29293.0$, & $(2529.6,2662.7$, \\
\hline$\hat{v}(\{2,3\})$ & $30757.7)$ & $2795.9)$ \\
\hline$\hat{v}(\{1,2,3\})$ & $(10677.9,11239.9$, & $(970.6,1021.7$, \\
& $11801.9)$ & $1072.8)$ \\
\hline
\end{tabular}

Table 1. Expected return of the players (unit: thousand dollars)

\begin{tabular}{|l|c|c|c|}
\hline \multicolumn{1}{|c|}{ Player } & Owner & Architect & Builder \\
\hline Budget cost & 149847.9 & 8847.9 & 141000.0 \\
\hline Average industry profit (\%) & 11.10 & 2.50 & 7.09 \\
\hline Expected return with the average profit rate & 16633.1 & 221.2 & 9996.9 \\
\hline Expected return with fuzzy value & $(15801.5,16633.1,17464.8)$ & $(210.1,221.2,232.3)$ & $(9497.1,9996.9,10496.8)$ \\
\hline
\end{tabular}




\subsection{Calculating the optimal allocation value of the players in the alliance}

According to the formula $\tilde{v}(S)(\alpha)=\left[v^{L}(S), v^{R}(S)\right]$ mentioned in Section 2.3, $\sum_{S \subseteq N: i \in S} w(S) v^{L}(S)(\alpha)(i=1,2,3)$ can be calculated as follows:

$$
\begin{aligned}
& \sum_{S \subseteq N: 1 \in S} \omega(S) v^{L}(S)(\alpha)=\frac{1}{6} \tilde{v}(1,2)+\frac{1}{6} \tilde{v}(1,3)+ \\
& \frac{1}{4} \tilde{v}(1,2,3)=1963.9+103.4 \alpha ; \\
& \sum_{S \subseteq N: 2 \in S} \omega(S) v^{L}(S)(\alpha)=\frac{1}{6} \tilde{v}(1,2)+\frac{1}{6} \tilde{v}(2,3)+ \\
& \frac{1}{4} \tilde{v}(1,2,3)=1704.0+89.7 \alpha ; \\
& \sum_{S \subseteq N: 3 \in S} \omega(S) v^{L}(S)(\alpha)=\frac{1}{6} \tilde{v}(1,3)+\frac{1}{6} \tilde{v}(2,3)+ \\
& \frac{1}{4} \tilde{v}(1,2,3)=1858.8+97.8 \alpha .
\end{aligned}
$$

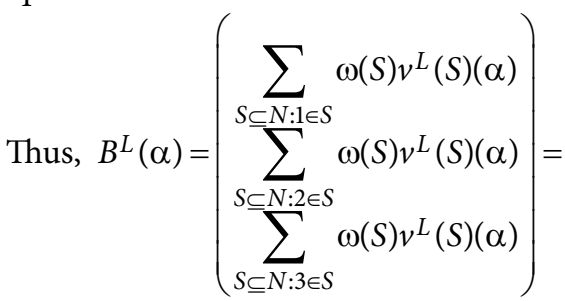$$
\left(\begin{array}{c}
1963.9+103.4 \alpha \\
1704.0+89.7 \alpha \\
1858.8+97.8 \alpha
\end{array}\right)
$$

Equation (12) indicates the following:

$$
\begin{aligned}
& x^{L}(\alpha)=A^{-1} B^{L}(\alpha)= \\
& \left(\begin{array}{ccc}
3 / 8 & -1 / 8 & -1 / 8 \\
-1 / 8 & 3 / 8 & -1 / 8 \\
-1 / 8 & -1 / 8 & 3 / 8
\end{array}\right)\left(\begin{array}{c}
9232.3+485.9 \alpha \\
7673.3+403.9 \alpha \\
8601.9+452.7 \alpha
\end{array}\right)= \\
& \left(\begin{array}{c}
1427.5+75.1 \alpha \\
648.5+34.1 \alpha \\
111.28+58.5 \alpha
\end{array}\right) .
\end{aligned}
$$

Likewise, we can determine that:

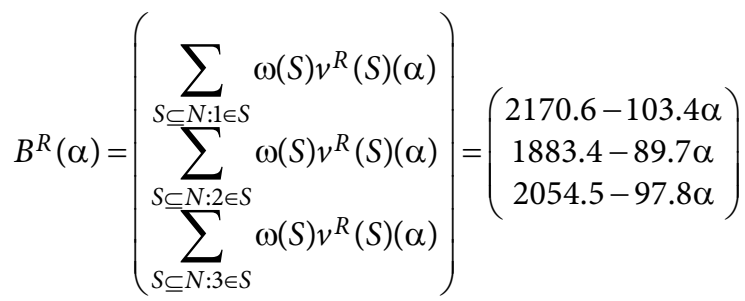

and $x^{R}(\alpha)=A^{-1} B^{R}(\alpha)=$

$$
\left(\begin{array}{ccc}
\frac{26}{9} & -\frac{10}{9} & -\frac{10}{9} \\
-\frac{10}{9} & \frac{26}{9} & -\frac{10}{9} \\
-\frac{10}{9} & -\frac{10}{9} & \frac{26}{9}
\end{array}\right)\left(\begin{array}{c}
1895.2-90.2 \alpha \\
746.5-35.5 \alpha \\
1430.7-68.1 \alpha
\end{array}\right)=
$$

$$
\left(\begin{array}{c}
1553.6-50.8 \alpha \\
740.6-58.5 \alpha \\
1254.0-83.0 \alpha
\end{array}\right)
$$

Therefore, according to $x_{i}(\alpha)=\left[x_{i}^{L}(\alpha), x_{i}^{R}(\alpha)\right](i=1$, $2 \ldots, n)$, the player's estimate of the TFN-type $\alpha$-cut set can be obtained:

$$
\begin{aligned}
& x_{1}(\alpha)=(1714.7+90.2 \alpha, 1895.2-90.2 \alpha) ; \\
& x_{2}(\alpha)=(675.4+35.5 \alpha, 746.5-35.5 \alpha) ; \\
& x_{3}(\alpha)=(1294.4+68.1 \alpha, 1430.7-68.1 \alpha) .
\end{aligned}
$$

According to the expression of the fuzzy set, $x_{i}=\left(x_{i}^{l}, x_{i}^{m}, x_{i}^{r}\right)=\left(x_{i}^{L}(0), x_{i}^{L}(1), x_{i}^{R}(0)\right)=\left(x_{i}^{L}(0), x_{i}^{R}(1)\right.$, $\left.x_{i}^{R}(0)\right)$. The player's estimated profit of TFN-type $\alpha$-cut set can be expressed as $x_{1}=\left(x_{1}^{l}, x_{1}^{m}, x_{1}^{r}\right)=\left(x_{1}^{L}(0), x_{1}^{L}(1)\right.$, $\left.x_{1}^{R}(0)\right)=(1714.7,1804.9,1895.2)$. In the same way, $x_{2}=$ $\left(x_{2}^{l}, x_{2}^{m}, x_{2}^{r}\right)=(675.4,710.9,746.5), x_{3}=\left(x_{3}^{l}, x_{3}^{m}, x_{3}^{r}\right)=$ $(1294.4,1362.6,1430.7)$. The calculation results are shown in Figure 2.

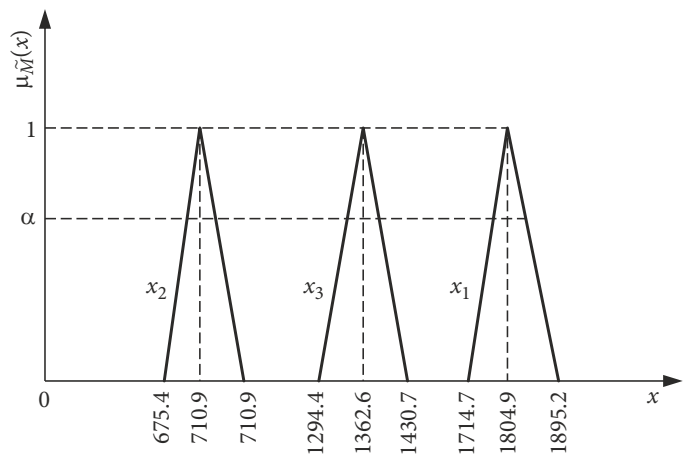

Figure 2. The TFN-typed imputations of players 1, 2 and 3

The results show that the profits obtained for the three players in the cooperative game have not been all distributed, that is, there is profits generated by the cooperation still have a surplus. Although this allocation scheme is certain fairness and rationality for the players, it is not the optimal allocation scheme and does not reach Pareto optimality.

As mentioned above, the efficiency condition is not considered in the above-mentioned player's estimated profit of the TFN-type $\alpha$-cut set. Therefore, the results are improved using Eqn (14) to adjust the distribution scheme.

In this case, $v^{L}(N)(\alpha)-\sum_{i=1}^{3} x_{i}^{L}(\alpha)$ and $v^{R}(N)(\alpha)-\sum_{i=1}^{3} x_{i}^{R}(\alpha)$ are calculated as follows:

$$
v^{L}(N)(\alpha)-\sum_{i=1}^{3} x_{i}^{L}(\alpha)=
$$

$$
5370.2 \alpha+5101.7(1-\alpha)-(1714.7+90.2 \alpha+675.4+
$$$$
35.5 \alpha+1294.4+74.6 \alpha)=1417.2+74.6 \alpha \text {; }
$$

$v^{R}(N)(\alpha)-\sum_{i=1}^{3} x_{i}^{R}(\alpha)=$

$5370.2 \alpha+5638.8(1-\alpha)-(1895.2-90.2 \alpha+746.5-$ 


$$
\begin{aligned}
& 35.5 \alpha+1430.7-68.1 \alpha)=1566.5-74.8 \alpha . \\
& x^{L *}(\alpha)=x^{L}(\alpha)+\frac{1}{3}\left(v^{L}(N)(\alpha)-\sum_{i=1}^{3} x_{i}^{L}(\alpha)\right) e= \\
& \left(\begin{array}{c}
1714.7+90.2 \alpha \\
675.4+35.5 \alpha \\
1294.4+74.6 \alpha
\end{array}\right)+\frac{1}{3}(1417.2+74.6 \alpha)\left(\begin{array}{l}
1 \\
1 \\
1
\end{array}\right)= \\
& \left(\begin{array}{c}
2187.1+113.1 \alpha \\
1147.8+60.4 \alpha \\
1766.8+93.0 \alpha
\end{array}\right) ; \\
& x^{R *}(\alpha)=x^{R}(\alpha)+\frac{1}{3}\left(v^{R}(N)(\alpha)-\right. \\
& \left.\sum_{i=1}^{3} x_{i}^{R}(\alpha)\right) e=\left(\begin{array}{c}
1895.2-90.2 \alpha \\
746.5-35.5 \alpha \\
1430.7-68.1 \alpha
\end{array}\right)+ \\
& \frac{1}{3}(1566.5-74.8 \alpha)\left(\begin{array}{l}
1 \\
1 \\
1
\end{array}\right)=\left(\begin{array}{c}
2417.3-115.2 \alpha \\
1268.6-60.5 \alpha \\
1952.8-93.0 \alpha
\end{array}\right) .
\end{aligned}
$$

At this point, the optimal distribution scheme satisfying the efficiency of all players has been obtained. The TFN-typed profit of player 1 in the grand alliance $N$ is expressed as

$$
x_{1}^{*}=\left(x_{1}^{l *}, x_{1}^{m *}, x_{1}^{r *}\right)=\left(x_{1}^{L *}(0), x_{1}^{L *}(1), x_{1}^{R *}(0)\right)=
$$

(2187.1, 2302.2, 2417.3);

$$
x_{2}^{*}=\left(x_{2}^{l *}, x_{2}^{m *}, x_{2}^{r *}\right)=\left(x_{2}^{L *}(0), x_{2}^{L *}(1), x_{2}^{R *}(0)\right)=
$$

(1147.8, 1208.2, 1268.6);

$$
x_{3}^{*}=\left(x_{3}^{l *}, x_{3}^{m *}, x_{3}^{r *}\right)=\left(x_{3}^{L *}(0), x_{3}^{L *}(1), x_{3}^{R *}(0)\right)=
$$

$(1766.8,1859.8,1952.8)$.

The calculation results are shown in Figure 3.

After considering the efficiency, the profit distribution of the three players in the TFN type is more

reasonable than the previous one. The lower limit, the mean limit, and the upper limit of the sum of the three player's allocation results are:

$$
\begin{aligned}
& 2187.1+1147.8+1766.8=5101.7 ; \\
& 2302.2+1208.2+1859.8=5370.2 ; \\
& 2417.3+1268.6+1952.8=5638.8 .
\end{aligned}
$$

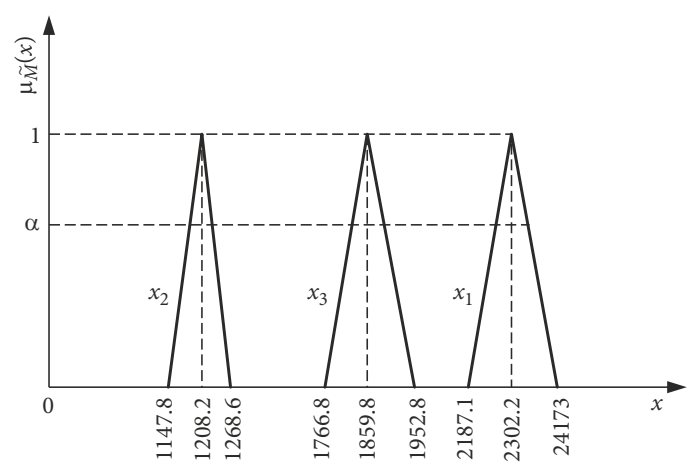

Figure 3. The optimal TFN-typed imputations of the players considering efficiency
Figure 3 shows that the expected distributable profit $\hat{v}$ $(\{1,2,3\})$ of the core players is consistent with the expected profit $\hat{v}(\{1,2,3\})=(5101.7,5370.2,5638.8)$. In other words, the payoff vector $x^{*}=\left(x_{1}^{*}, x_{2}^{*}, x_{3}^{*}\right)$ obtained from the allocation method is efficient.

\section{Discussion}

The computational results gained from the quadratic programming model that considers the efficiency indicates that all the participants in the cooperative game receive a fair profit distribution. We use player 2 as an example to analyze the profit in different alliance situations. When player 2 does not participate in the alliance or the project, their profit is 0 . When they join the grand cooperative alliance, the lower limit and the upper limit of the sum of the three players' profits are $\$ 1147.8$ and $\$ 1268.6$, respectively. Moreover, the most likely payoff for player 2 is $\$ 1208.2$. The change in the profit allocated by the grand cooperative alliance $N$ to players 1 and 3 is similar to that of player 2. In summary, player 2 will gain more imputation in the alliance game due to the transferable payoff. In other words, all participants in an alliance want to maximize their profits if they cooperate on the IPD project. As a consequence, the grand alliance $N$ is strong, and each construction enterprise shows great enthusiasm to participate.

Next, we compute the profit distribution of the alliance profits using the Shapley value (Shapley, 1953) to demonstrate the superiority of the proposed method.

As described in Section 3.2, for the fuzzy numbers $\tilde{a}=$ $\left(a^{l}, a^{m}, a^{r}\right)$, when $a^{l}=a^{m}=a^{r}$, the TFNs $\tilde{a}=\left(a^{l}, a^{m}, a^{r}\right)$ degenerate into real numbers. The fuzzy cooperative game becomes a crisp cooperative game. The fuzzy payoffs $\tilde{v}(S)$ are reduced to $v(\mathrm{~S})$.

In the crisp cooperative game $(N, v)$, there is a unique Shapley function $\varphi_{i}(v): P(X) \rightarrow \mathbb{R}$

$$
\phi_{i}(v)=\sum_{S \subseteq N: i \in S} \gamma_{n}^{s}[v(S)-v(S-\{i\})],
$$

where $\gamma_{n}^{s}=\frac{(n-s) !(s-1) !}{n !}$, with $s=|S|, i \in N$.

In this case, if the value of the imputation reaches a certain value, the fuzzy cooperative game becomes a crisp cooperative game. Furthermore, we use the classic Shapley value method to calculate the imputation of the owner, the architect, and the builder. The distributable profits of various alliances are shown in Table 3.

Table 3. Distributable profits of the Alliance in the crisp game (unit: thousand dollars)

\begin{tabular}{|l|c|c|c|c|}
\hline \multicolumn{1}{|c|}{ Alliance } & $v(\{1,2\})$ & $v(\{1,3\})$ & $v(\{2,3\})$ & $v(\{1,2,3\})$ \\
\hline $\begin{array}{l}\text { Expected earnings } \\
\text { of alliance }\end{array}$ & 18539.7 & 29293.0 & 11239.9 & 32221.5 \\
\hline $\begin{array}{l}\text { Alliance } \\
\text { distributable profit }\end{array}$ & 1685.3 & 2662.7 & 1021.7 & 5370.2 \\
\hline
\end{tabular}


The Shapley values of players are calculated using Eqn (15) (Table 4).

Consequently, $\varphi_{1}(v)=\sum_{S \subseteq N: i \in S} \gamma_{n}^{s}[v(S)-v(S-\{1\})]=0+$ $280.9+443.8+1449.5=2174.2$ thousand dollars. Similarly, the profit distributed by the builder is $\$ 1,842.4$. The three core players split the proceeds at $\$ 5,370.2$. Thus, the Shapley vector is $\varphi(v)=\left(\varphi_{1}(v),\left(\varphi_{2}(v),\left(\varphi_{3}(v)\right)=(2174.2\right.\right.$, $1353.7,1842.4)$. According to the Shapley value, the profits are distributed among the owner, the architect, and the builder in different crisp alliance situations. The details of the profit distribution are listed in Table 5.

In these alliances, if a player earns more profits by participating in the grand alliance, it will join the alliance. Otherwise, the player operates as an individual. A comparison of the results for the crisp grand alliance and the TFN-type fuzzy alliance is provided in Table 6.

Analyzing Table 6, there are three characteristics as follows:

(1) Comparison of the profits in the crisp grand alliance and the TFN-type fuzzy alliance that considers efficiency. In both cases, the distributable profit is $\$ 5,370.3$. Due to the importance of the alliance, the profit of the owner is higher in the crisp grand than in the fuzzy alliance. The architect's profit has decreased, and the builder' profit has changed slightly little. The owners are the most important players in the alliance.

(2) Comparison of the TFN-type fuzzy value with and without considering efficiency. The difference be-

Table 4. Shapley value of the owner (unit: thousand dollars)

\begin{tabular}{|l|c|c|c|c|}
\hline \multicolumn{1}{|c|}{ Alliance } & $\{1,2\}$ & $\{1,3\}$ & $\{2,3\}$ & $\{1,2,3\}$ \\
\hline$v(\{S\})$ & 0 & 1685.3 & 2662.7 & 5370.2 \\
\hline$v(\{S\}-1)$ & 0 & 0 & 0 & 1021.7 \\
\hline$v(\{S\})-v(\{S\}-1)$ & 0 & 1685.3 & 2662.7 & 4348.5 \\
\hline$\gamma^{s}$ & $1 / 3$ & $1 / 6$ & $1 / 6$ & $1 / 3$ \\
\hline$\gamma^{S}[v(S)-v(S-\{i\})]$ & 0 & 280.9 & 443.8 & 1449.5 \\
\hline
\end{tabular}

Table 5. Strategies of the profit distribution among the players in different crisp alliance combinations (unit: thousand dollars)

\begin{tabular}{|l|c|c|c|}
\hline \multicolumn{1}{|c|}{ Alliance } & Owner & Architect & Builder \\
\hline$\varphi_{i}(v)(\{1,2\})$ & 1038.6 & 646.7 & 0 \\
\hline$\varphi_{i}(v)(\{1,3\})$ & 1441.3 & 0 & 1221.4 \\
\hline$\varphi_{i}(v)(\{2,3\})$ & 0 & 432.7 & 589.0 \\
\hline$\varphi_{i}(v)(\{1,2,3\})$ & 2174.2 & 1353.7 & 1842.4 \\
\hline
\end{tabular}

tween the payoff values calculated by the two algorithms is relatively large. The reason is that the profit generated by the cooperation is not entirely distributed, and there is a surplus because only the fair distribution of the profit among the members is considered in the TFN-type fuzzy algorithm. In the modified model of the TFN-type value, efficiency and fairness are considered. Additionally, Eqn (3) indicates that this model is a Pareto optimal distribution scheme.

(3) Comparison of the crisp grand alliance and the modified model of the TFN-type value. The Shapley value for determining the profit distribution is based on the consistent risk and contribution of all parties, which is too idealistic. Furthermore, IPD projects are characterized by long duration, high complexity, and uncontrollable factors. As a result, the profit distribution is difficult to define accurately. It is more reasonable to regard the project alliance as a fuzzy cooperative alliance. The profit obtained from the TFN $\alpha$-cut set method is closer to engineering practice than the crisp grand alliance. Therefore, the proposed methodology is practical and applicable to the situation.

In this study, the first step in the proposed methodology provides the $\alpha$-cut set and the weights of alliance that expresses the pre profit distribution of each player in the TFN-type alliance, ensuring a fair profit distribution. The results of this step are used as inputs for the next step, which provides the fuzzy payoffs of the participants. The pre profit distribution to the three core players is represented in Figure 2. Then, the profit is redistributed considering the efficiency of the cooperative game in the modified model. The optimization model defined in Eqn (14) is used to calculate the optimal quadratic programming model in the different fuzzy alliances and the value of the fuzzy alliance. Furthermore, Eqn (15) is used to obtain the final payoffs of the players in the fuzzy cooperative game.

Of course, there are other researchers who have studied profit distribution in other methodologies. In this paper, one of the most frequently cited articles in the past five years will be selected for comparison. Further, we compared the research results of this paper with those of Teng et al. (2017), who also investigated the profit distribution in IPD projects using a cooperative game approach. However, our research methods were different. Teng et al. (2017) regarded the alliance as a crisp alliance, and they used the Shapley value method to preliminarily distribute the profit. The Shapley value was used as the solution to the cooperative game and to determine the

Table 6. Compared with the imputations for the crisp grand alliance and the TFN-type fuzzy alliance (unit: thousand dollars)

\begin{tabular}{|l|c|c|c|}
\hline \multicolumn{1}{|c|}{ Player } & The value in crisp grand alliance & The TFN-type fuzzy value & The TFN-type fuzzy value from the modified model \\
\hline Owner & 2174.2 & $(1714.7,1804.9,1895.2)$ & $(2187.1,2302.2,2417.3)$ \\
\hline Architect & 1353.7 & $(675.4,710.9,746.5)$ & $(1147.8,1208.2,1268.6)$ \\
\hline Builder & 1842.4 & $(1294.4,1362.6,1430.7)$ & $(1766.8,1859.8,1952.8)$ \\
\hline
\end{tabular}


profit distribution among the stakeholders. In addition, Teng et al. (2017) adopted risk-sharing of the four stakeholders to modify the Shapley value.

Our proposed methodology considers the alliance weights and efficiency in fuzzy cooperative games and maximizes the distribution of benefits to the participants in a fair and reasonable manner. Therefore, it can be concluded that this methodology is suitable for the profit distribution because efficiency and equity are taken into account. In addition, the method has a straightforward calculation, easy implementation, and closely reflects actual conditions.

\section{Conclusions}

The method presented in this paper represents the first attempt to use the quadratic programming model and method to solve the profit distribution of IPD projects. We establish the fuzzy payoff functions for the TFNs and $\alpha$-cut sets expression. Due to the asymmetry of the capability of participants, the introduction of the weight of the alliance can better express the information of each participant and make the IPD profit distribution more equitable. By considering the effectiveness of cooperative game, the profit distribution can be complete without surplus. The satisfaction of the IPD project participants can be improved and the stability of the IPD alliance and the work enthusiasm of all participants can be increased. We anticipate this method to be useful for virtual enterprises, supply chain management and other issues related to profit distribution. The study provides a new perspective that enriches the theoretical research of profit distribution in IPD projects and offers theoretical guidance for practical applications and the promotion of IPD in the AEC industry, promoting the efficient, fair, and healthy development of the AEC industry.

The theoretical research in this paper has several limitations. First, we made several assumptions on the profit growth of cooperative alliance to simplify the model. At present, there is no widely accepted data on growth rates. The topic that requires further research. Second, this research used the basic assumption of a convex cooperative game. The concepts of the TFNs and the $\alpha$-cut sets rely on this assumption. Convexity is a sufficient condition for the fuzzy strong core in the proposed methodology. A convex cooperative game is a special case of a cooperative game. It is the main limitation of the proposed methodology. Third, the method for determining the income distribution depends on the contract. IPD includes three types of contracts: transitional contracts, relational contracts, and Single Purpose Entities (SPE). The IFOA contract in this study is a tri-party contract, and the profit distribution of the IPD under other contract conditions is not considered. Future studies should focus on the impact of SPE on the profit distribution in IPD projects. Moreover, weighted triangular approximation, a generalized symmetric TFN approach to solving the profit distribution in IPD projects, should be considered in future studies.

\section{Acknowledgements}

The authors thank Kang Du for technical help.

\section{Funding}

This research was funded by the Key Projects of Social Science Research of Department of Education of Hubei Province of China (No. 20D914).

\section{Disclosure statement}

The authors have declared no conflicts of interest.

\section{Author contributions}

S. W. Guo and J. W. Wang conceived and designed the study. S. W. Guo wrote the paper. S. W. Guo and J. W. Wang reviewed and edited the manuscript. All authors read and approved the manuscript.

\section{References}

Abraham, S., \& Punniyamoorthy, M. (2021). A fuzzy approach using asymmetrical triangular distribution in a two-person zero-sum game for a multi-criteria decision-making problem. Quantum Machine Intelligence, 3(1), 20.

https://doi.org/10.1007/s42484-021-00044-y

American Institute of Architects. (2010). Integrated project delivery: Case studies. California Council.

Alparslan-Gök, S. Z., Miquel, S., \& Tijs, S. (2009). Cooperation under interval uncertainty. Mathematical Methods of Operations Research, 69, 99-109.

https://doi.org/10.1007/s00186-008-0211-3

Ashcraft, H. W. (2012). The IPD framework. Hanson Bridgett.

Babbar, N., Kumar, A., \& Bansal, A. (2013). Solving fully fuzzy linear system with arbitrary triangular fuzzy numbers. Soft Computing, 17(4), 691-702.

https://doi.org/10.1007/s00500-012-0941-2

Boodai, F. J. (2014). Achieving construction project success through integration in the project delivery system from an owner's perspective [Doctoral dissertation]. The University of Wisconsin, Madison.

Construction Management Association of America. (2010). Managing integrated project delivery. https://www.leanconstruction.org/wp-content/uploads/2016/02/CMAA_Managing_Integrated_Project_Delivery_1.pdf

United Kingdom's Office of Government Commerce. (2007). Achieving excellence in construction Procurement Guide 05: The integrated project team: Team working and partnering.

Dubois, D., \& Prade, H. (1980). Fuzzy sets and systems: Theory and applications. Academic Press.

Eissa, R., Eid, M. S., \& Elbeltagi, E. (2021). Conceptual profit allocation framework for construction joint ventures: Shapley value approach. Journal of Management in Engineering, 37(3), 04021016. https://doi.org/10.1061/(ASCE)ME.1943-5479.0000911

Ernst and Young. (2014). Spotlight on oil and gas megaprojects (No. DW0426). EYGM Limited, EYG.

Han, T., \& Li, D. (2016). Shapley value method of profit allocation for cooperative enterprises with intuitionistic fuzzy coalitions. Journal of Systems Science and Mathematical Sciences, $36,719-727$. 
Jene, S., \& Zelewski, S. (2014). Practical application of cooperative solution concepts for distribution problems: an analysis of selected game theoretic solution concepts from an economic point of view. International Journal of Mathematics, Game Theory, and Algebra, 23(1), 19-37.

Jia, N. X., \& Yokoyama, I. L. (2003). Profit allocation of independent power producers based on cooperative game theory. Electrical Power and Energy Systems, 25, 633-641. https://doi.org/10.1016/S0142-0615(02)00180-1

Khalfan, M. M. A., \& McDermott, P. (2006). Innovating for supply chain integration within construction. Construction Innovation, 6, 143-157. https://doi.org/10.1108/14714170610710695

Li, D.-F. (2012). A fast approach to compute fuzzy values of matrix games with payoffs of triangular fuzzy numbers. European Journal of Operational Research, 223(2), 421-429. https://doi.org/10.1016/j.ejor.2012.06.020

Lin, L., \& Wang, H. (2019). Dynamic incentive model of knowledge sharing in construction project team based on differential game. Journal of the Operational Research Society, 70, 2084-2096. https://doi.org/10.1080/01605682.2018.1516177

Liu, Q., \& Cheng, Z. (2020). Research on profit distribution of construction project dynamic alliance under integrated project delivery mode. Value Engineering, 39, 58-61.

Long, Y., Peng, J., \& Iwamura, K. (2009). Uncertain equilibrium analysis on profits distribution between partner firms in competitive strategic alliances. Soft Computing, 13(2), 203-208. https://doi.org/10.1007/s00500-008-0302-3

Meade, L. M., \& Lilesa, D. H. (1997). Justifying strategic alliances and partnering: a prerequisite for virtual enterprising. Omega, 25(1), 29-42. https://doi.org/10.1016/S0305-0483(96)00034-5

Moore, R. E. (1979). Methods and applications of interval analysis. DBLP. https://doi.org/10.1137/1.9781611970906

Myerson, R. B. (1997). Game theory: Analysis of conflict. Harvard University Press.

Pishdad-Bozorgi, P., \& Srivastava, D. (2018). Assessment of integrated project delivery (IPD) risk and reward sharing strategies from the standpoint of collaboration: A game theory approach. In Proceedings of Construction Research Congress 2018: Construction Project Management (pp. 196-206). ASCE. https://doi.org/10.1061/9780784481271.020

Shapley, L. S. (1953). A value for n-persons games. Annals of Mathematics Studies, 28(7), 307-318. https://doi.org/10.1515/9781400881970-018

Su, D., \& Yang, J. (2018). Distribution model of fuzzy graph cooperative games with restricted alliance and its application. Journal of FuZhou University (Natural Science Edition), 46, 458-465.

$\mathrm{Su}, \mathrm{S}$. (2020). Research on triangular fuzzy number type entrepreneurial team many persons income distribution cooperation game based on satisfactory degree. Mathematics in Practice and Theory, 50(1), 1-8.

Teng, Y., Li, X., Wu, P., \& Wang, X. (2017). Using Cooperative game theory to determine profit distribution in IPD projects. International Journal of Construction Management, 19, 32-45. https://doi.org/10.1018/15623599.2017.1358075

Wang, R., \& Yuan, Z. (2019). Dynamic distribution of cooperative profit mechanism in EPC projects considering satisfaction of all parties. Journal of Engineering Management, 33, 13-18. https://doi.org/10.13991/j.cnki.jem.2019.02.003

Ye, Y., Li, D., \& Yu, G. (2019). A joint replenishment model with demands represented by triangular fuzzy numbers and its cost allocation method. Journal of Systems Science and Complexity, 39, 1142-1158.
Yu, X., \& Zhang, Q. (2019). Core for game with fuzzy generalized triangular payoff value. International Journal of Uncertainty Fuzziness and Knowledge-Based Systems, 27(5), 789-813. https://doi.org/10.1142/S0218488519500351

Zhao, W. J., \& Liu, J. C. (2018). Triangular fuzzy number-typed fuzzy cooperative games and their application to rural e-commerce regional cooperation and profit sharing. Symmetry, 10(12), 699. https://doi.org/10.3390/sym10120699 